\title{
Efficient Routing Technique in Heterogeneous Wireless Network
}

\author{
Alisha Dua \\ M.Tech Scholar, DCSA \\ Maharishi Dayanand \\ University, Rohtak
}

\author{
Sandeep Dalal, PhD \\ Asst. Professor, DCSA \\ Maharishi Dayanand \\ University, Rohtak
}

\author{
Kamna Solanki \\ Asst. Professor, UIET \\ Maharishi Dayanand \\ University, Rohtak
}

\begin{abstract}
The paper discusses an efficient algorithm for the routing in the heterogeneous wireless network. The advancement in the technology and introduction of LTE needs the interfacing with different types of environment. Due to different characteristics of each network the performance of the homogeneous routing protocols degrades. The paper modifies the AODV routing and introduces the HAODV for the heterogeneous environment. In the HAODV packet format, one field named rc i.e. routing cost is added to evaluate the routing cost. The rc is calculated based on the link quality and the traffic demand. The performance analysis is done by using various parameters i.e. PDR, E2Edelay and the throughput. The simulation result shows that the algorithm is better than the existing algorithms.
\end{abstract}

\section{Keywords}

Routing cost, link quality, traffic demand, AODV, RREQ

\section{INTRODUCTION}

The advancement in the wireless communication network technology leads to the heterogeneity i.e. access of different network at one terminal. The mobile device must be able to use these technologies simultaneously [1] [4]. The different bandwidth and other different attributes of different network need them to be operated independently. The routing among such networks is key issue as the performance degrades due to different features. This paper discusses the modified AODV i.e. HAODV for the routing in heterogeneous network. The algorithm discussed in the paper is generalized while the implementation is carried out with the two types of nodes i.e. WIFI, WIMAX. The rest paper is divided as follow: section 2 describes the AODV routing, section discuss the related work. Then the HAODV is presented in section 4. The implementation and results are discussed in section 5 .

\section{AODV}

AODV routing protocol is a table driven routing protocol that manages the routing table to find a route. It is designed for mobile ad hoc networks with tens to thousands number of mobile nodes .It is a reactive routing protocol that waits for requests before attempting to find the most optimal route from one node(source node) to send messages to another node (destination). The most optimal route is determined by the distance or the number of hops between nodes. Sequence numbers at destination is used for loop freedom.

\subsection{Message Type in AODV}

The AODV protocol uses 4 types of messages to discover, establish and maintain the routes. Route Request (RREQ), Route Reply (RREP), Route Error (RERR) and HELLO messages.

\subsubsection{Route Request (RREQ)}

The source node broadcasts a RREQ packet to find a route to the destination. Route request packet (RREQ) format is given in table

Table1. RREQ packet format

\begin{tabular}{|c|c|c|c|}
\hline Type & $\mathrm{J}|\mathrm{R}| \mathrm{G}|\mathrm{D}| \mathrm{U}$ & Reserved & $\begin{array}{c}\text { Hop } \\
\text { Count }\end{array}$ \\
\hline \multicolumn{4}{|c|}{ RREQ_ID } \\
\hline \multicolumn{4}{|c|}{ DESTINATION IP_ADDRESS } \\
\hline \multicolumn{4}{|c|}{ DESTINATION SEQUENCE_NUMBER } \\
\hline \multicolumn{4}{|c|}{ ORIGINATOR IP_ADDRESS } \\
\hline \multicolumn{4}{|c|}{ ORIGINATOR SEQUENCE_NUMBER } \\
\hline
\end{tabular}

\subsubsection{Route Reply (RREP)}

Any node having a fresh route cans unicast the RREP to the RREQ originator node. The packet format of RREP is given in table:

Table 2. RREP packet format

\begin{tabular}{|c|c|c|c|c|}
\hline Type & R|A & Reserved & $\begin{array}{c}\text { Prefix } \\
\text { Size }\end{array}$ & $\begin{array}{c}\text { Hop } \\
\text { Count }\end{array}$ \\
\hline \multicolumn{3}{|c|}{ DESTINATION IP_ADDRESS } \\
\hline \multicolumn{3}{|c|}{ DESTINATION SEQUENCE_NUMBER } \\
\hline \multicolumn{3}{|c|}{ ORIGINATOR IP_ADDRESS } \\
\hline \multicolumn{3}{|c|}{ LIFETIME }
\end{tabular}

\subsubsection{Hello Messages}

Nodes maintain the route by monitoring the link status of next hops in active routes by periodically sending the HELLO messages. HELLO message are not broadcasted to the network because these messages has TTL value 1 .

\subsubsection{Route Error Message (RERR)}

When link break in an active route is detected, a RERR message is sent to other nodes. The RERR has indication about all the routes that are using that broken link. RERR message contains:

Table 1.3: RERR packet format

\begin{tabular}{|c|c|c|c|}
\hline Type & $\mathrm{N}$ & Reserved & Discount \\
\hline & \multicolumn{3}{|c|}{$\begin{array}{l}\text { UNREACHABLE DESTINATION IP } \\
\text { ADDRESS(1) }\end{array}$} \\
\hline & & \multicolumn{2}{|c|}{$\begin{array}{l}\text { UNREACHABLE DESTINATION } \\
\text { SEQUENCE NUMBER(1) }\end{array}$} \\
\hline & & \multicolumn{2}{|c|}{$\begin{array}{l}\text { UNREACHABLE DESTINATION IP } \\
\text { ADDRESS(IF NEEDED) }\end{array}$} \\
\hline & & \multicolumn{2}{|c|}{$\begin{array}{l}\text { UNREACHABLE DESTINATION } \\
\text { SEQUENCE NUMBER(IF NEEDED) }\end{array}$} \\
\hline
\end{tabular}


Each node in network keeps a precursor list which contains the IP address for each its neighbors that are likely to use it as next hop towards each destination.

\subsection{Sequence Numbers}

Sequence numbers are used in AODV to ensure the route freshness. In AODV sequence numbers are updated by the source node when it generates a new route request to a destination or by the destination node while it sends a route reply. In case the source node receives more than one route reply messages for a requested destination, the route to the destination node with greatest sequence number is selected .It ensures the freshness of selected route. If there are multiple route reply messages with same sequence number then source node will select the route where hop count is less.

\subsection{Routing Table Management}

Every node in AODV maintains a routing table. AODV does not maintain the entire route to the destination. Each node only maintains the next hop information, this reduces processing and storage overhead for maintaining routes. A node update the routing table when it receives a control packet, the routing table will be checked for existence of entry for that destination. If no matching entry for that destination is found, a new table entry will be created. If the routing table entry for the destination is present, then the sequence number for that destination will be updated if the control packet updates the sequence number for that destination if the packet has sequence number higher than the destination sequence number in the routing table. Routing table has nine fields as follows:

a. Destination IP Address

b. Destination Sequence number

c. Valid Destination Sequence Number flag

d. State and routing flags like valid, invalid, repairable ,under repair

e. Network interface

f. Hop Count (Source to Destination)

g. Next Hop

h. Precursor list

i. Lifetime ( route expiration/deletion time)

Along with the source and destination sequence numbers the Route Request Expiration Timer and Route Caching Timeout are used to determine a route is still active or not. The entries for nodes that are not on the source to destination route are considered invalid. A route stays valid only for route caching timeout all entries for routes not used for route caching timeout are invalidated. List of precursors is maintained in order to send the error local repair notifications when next hop link loss is found in a route. Precursor list contains the list neighbor nodes to which a node generates the route reply messages.

\subsection{Route Discovery}

While communication routes between nodes are valid, AODV does not play any role. When a source node does not have fresh enough route to the destination, it initiates a route discovery process for the destination node by broadcasting a RREQ message. A fresh route to destination is found when the RREQ reaches either to the destination or any intermediate node has fresh enough route to the destination.
Every intermediate node increments the hops count value in RREQ message by one. Any node which has a route with greater sequence number as compared to the sequence number in the RREQ message is considered as fresh route .Reverse route is maintained to send back the RREP to the originator of RREQ by maintaining the precursor list for the next hop from which a node receives a RREQ message. Every intermediate node when receives a RREQ message updates it destination sequence number if required. It reads the address of node from which it receives the RREQ from the RREQ message and update it with its own before forwarding it towards the destination. RREP message is unicasted to the source node over the reverse route. The source to destination route is established when the source node receive the RREP message. If the source received more than one RREP messages then the RREP message with greater sequence number is considered.

\subsection{Maintaining Local Connectivity}

To maintain the local connectivity among neighbor nodes Hello Messages are sent with TTL value set to 1 . Every node that is part of active route should send HELLO messages to ensure local connectivity. If a node does not send Hello message or any other packet for AALOWED_HELLO_LOSS * HELLO_INTERVAL milliseconds, the neighboring node will consider the link to that node is lost. HELLO packets are sent only when the neighboring nodes are not sending any packets.

\section{RELATED WORK}

The author of. [2] proposed a data aggregation technique that work on the wired as well on wireless interface to enhance the QOS. The paper [3] proposed a simple technique for handover in ATM based PCN network. The hybrid scheme enhances the QoS. Anthony. Authors of paper [5] present a architecture that uses the Earliest Delivery Path First to deliver the packet from source to destination within the deadline. The scheduling algorithm enables the multi-access applications. The author of [6] survey the existing control schemes for the $2 \mathrm{G}$ as well for $3 \mathrm{G}$ network. The author also give a design of control scheme for the 4G applications. The paper [7] studied the resource management within the network. The authors uses the priority based access to proper manager the resources.

The paper [8] proposed the system level Quality-of-Service (QoS) at the data link layer. This system level QoS is designed to guarantee the drop rate in wireless cellular networks. The author of paper [10] proposed a novel probability model to study the top redundancy level in terms of source redundancy and path redundancy. The paper. [11] presented, an energy-efficient scheme that performs bandwidth aggregation and supports real-time data-streaming services. These services are supported over asymmetric as well as on symmetric wireless links without any modifications in existing Internet infrastructure. The authors of paper [12] analyze routing protocol for MANET by using the PDF and delay through different scenarios that differentiate in the number of sources and pause time. The paper [13] proposed a simple and application layer Scheme that aggregates data which can be easily and dynamically transferred by using multiple links either parallelly or sequentially. They achieved $65 \%$ improvement over existing throughput as compared to existing application layer technique.

\section{HAODV}

The HAODV is the modified version of the AODV for the heterogeneous environment. The HAODV packet format has one extra field i.e. rc(routing cost). The routing cost is calculated as follow: 
The routing cost for heterogeneous environment is dependent upon the link quality and the traffic demand. The link quality of specific node and the target network must match for the efficient results. The DLQ i.e. difference in link quality is evaluated by the formulae given in (1)

$\mathrm{DLQ}=\mathrm{abs}(\operatorname{linkQ}($ node $)-\operatorname{linkQ}(\mathrm{tn}))$

The other factor the traffic demand, the rate of traffic flow through a node must match the traffic demand of the tn (target network) for the efficient performance. The DT i.e. difference if traffic is calculated by (2)

$\mathrm{DT}=\mathrm{abs}(\operatorname{tr}($ node $)-\operatorname{tr}(\operatorname{tn}))$

The routing cost can be calculated from the DLQ and DT by using equation (3)

$\mathrm{rc}=\mathrm{c} 1 *(\mathrm{DLQ} / \mathrm{linkQ}(\mathrm{tn}))+\mathrm{c} 2 *(\mathrm{DT} / \mathrm{tr}(\mathrm{tn}))$

The routing cost must be low for the efficient routing. The working steps can be easily understood by the following algorithm:

\section{Select $\mathrm{S}$ and $\mathrm{D}$}

2. Active_N=S

3. While Active_N != D Repeat steps 4 to

4. Brodacast from node Active_N.

5. The group of nodes(say $G$ ) at one hop from Active_N receive the RREQ

6. $\quad$ Current $=\mathrm{S}$

7. While current $!=\mathrm{D}$

8. $\mathrm{G}=$ Select nodes near to the boundary of range of current

9. Calculate the rc for each node in $G$ by using equation (3).

10. Forward the data to node with minimum rc.

11. Active $\mathrm{N}=\operatorname{minrc}(\mathrm{G})$

12. End while

13. Send RREP and transfer the data.

The above algorithm is an efficient algorithm for the transfer of data in any heterogeneous environment. The result analysis is done in next section.

\section{RESULTS AND DISCUSSION}

The algorithm is analyzed by using NS2 by using nodes of WIMAX and WI-FI types. The analysis is done by using following parameters.

\section{- $\quad$ Routing Overhead}

The total number of routing packets transmitted during the simulation i.e. the sum of all transmissions of routing packets sent during the simulation.

Calculated as Routing Overhead $=\Sigma$ Transmission of routing packets

\section{- $\quad$ Packet Delivery Ratio}

The ratio of the number of sent data packet to the destination. This shows the level of sent data to the destination.

$\sum$ Number of packet receive / $\sum$ Number of packet send

The better value of packet delivery ratio means the enhanced performance of the protocol.

\section{- $\quad$ End-to-end Delay}

The usual time taken by a data packet to reach in the destination. It also comprises the delay caused by the queue in data packet transmission and route discovery process. Only the data packets that successfully sent to destinations counted.

$\sum$ (arrive time - send time) / $\sum$ Number of connections The results are shown in the following graphs:
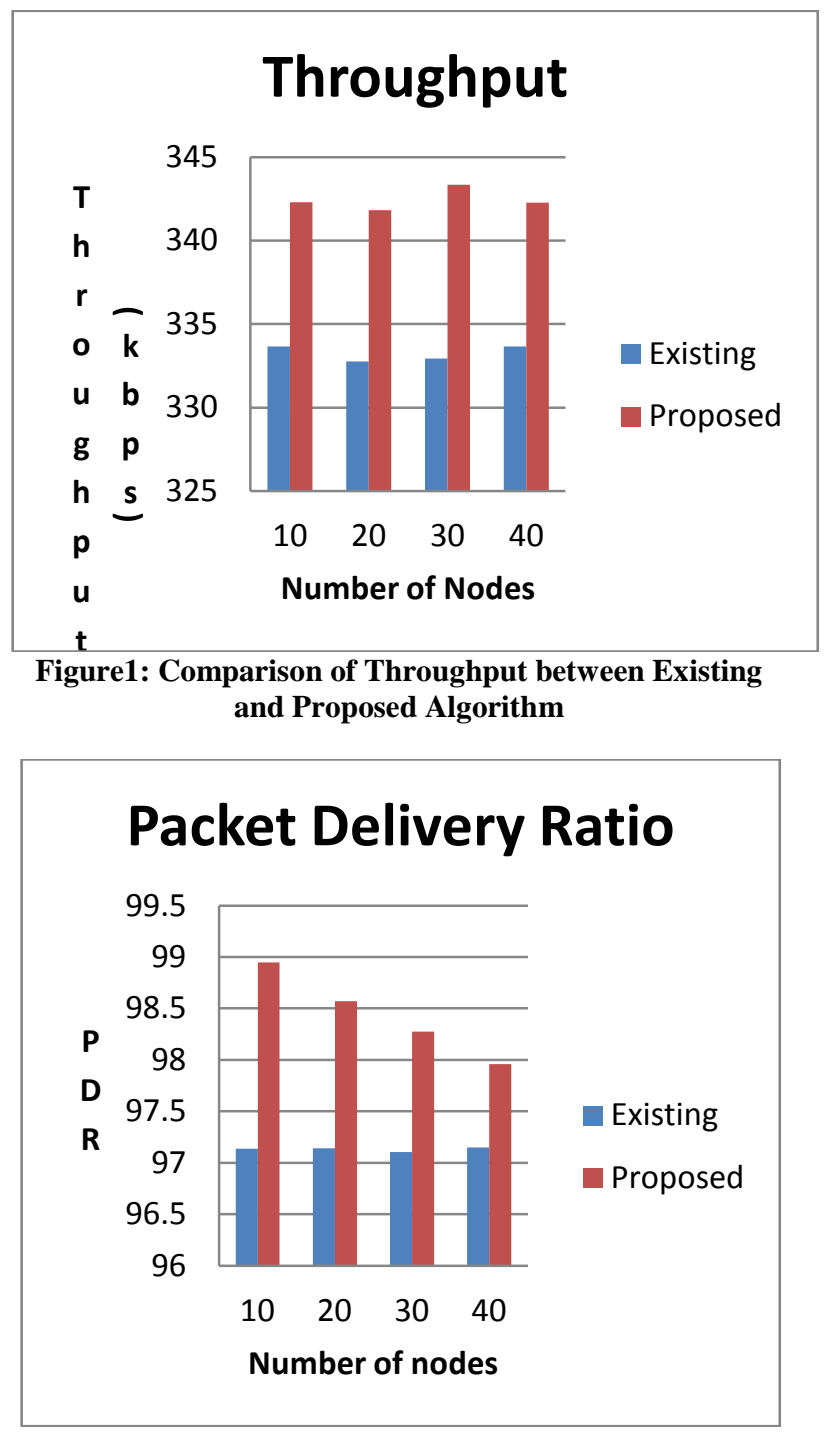

Figure 2: Comparison of Packet Delivery Ratio between Existing and Proposed Algorithm 


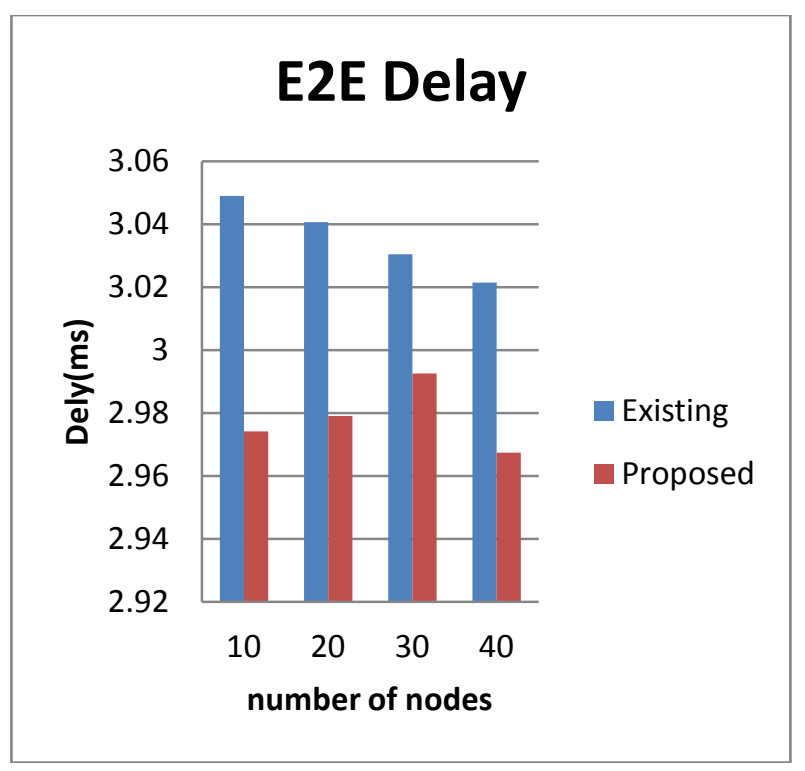

Figure 3: Comparison of E2 E Delay between Existing and Proposed Algorithm

The results can also be analyzed by varying the pause time as shown in the figure 4,5 and 6.

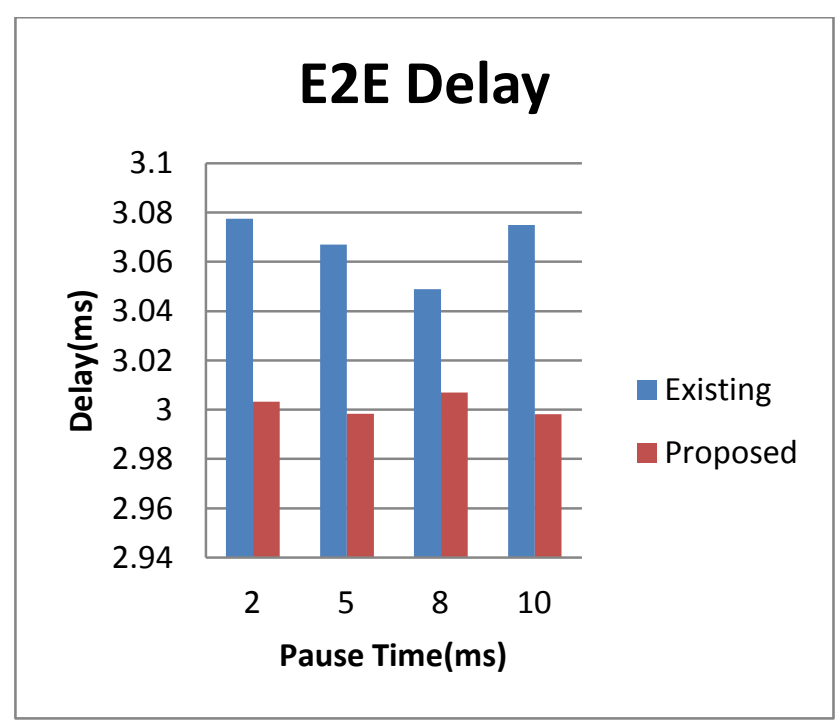

Figure 4: Comparison of E2 E Delay between Existing and Proposed Algorithm (pause time variation)

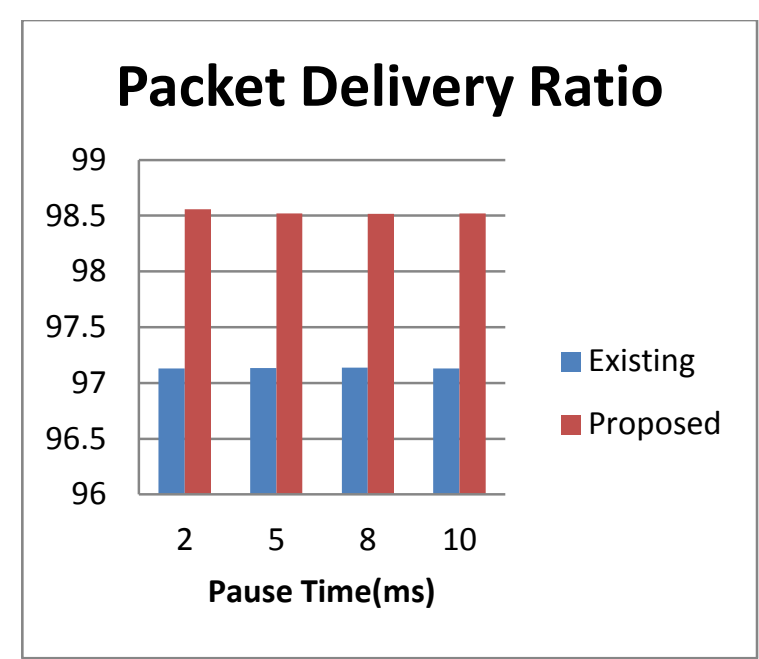

Figure 5: Comparison of Packet Delivery Ratio between Existing and Proposed Algorithm (pause time variation)

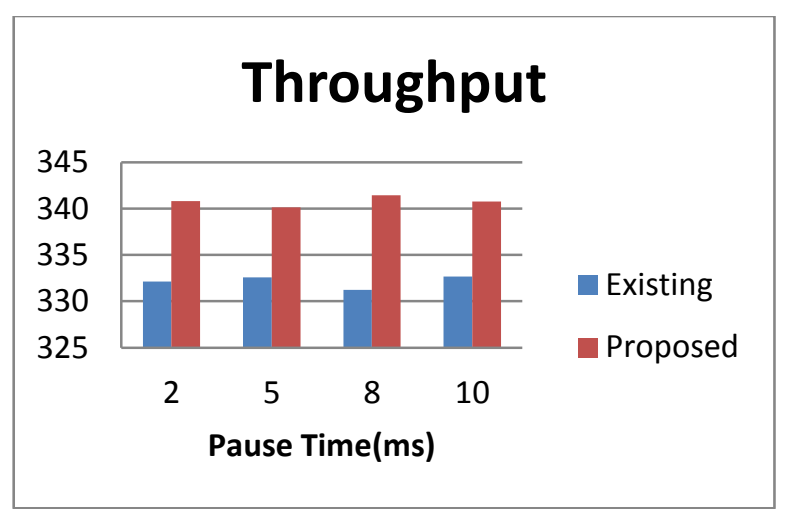

Figure6: Comparison of Throughput between Existing and Proposed Algorithm

The graphical and tabular comparison shows that the proposed algorithm is better than the existing algorithm in terms of various parameter i.e. PDR, Throughput and the E2Edelay. The proposed protocol increases the throughput decrease the E2Edelay. The increase in the PDR leads to the increase in the throughput. As the packet delivery ratio of the proposed protocol is better than the existing protocol so the quality of service of the proposed protocol is better than the existing protocol.

\section{CONCLUSION}

The paper proposes an efficient algorithm for the routing of data in heterogeneous network. The paper designs a routing protocol HAODV. The HAODV is the modified version of the AODV for the heterogeneous environment. The HAODV packet format has one extra field i.e. rc(routing cost). The rc is used for selection of nodes for routing. The analysis of the algorithm is done using the e2edelay, PDR and the throughput. Excellent performance of the proposed protocol is achieved by increasing throughput and decreasing end to end delay. In future the algorithm can be extended in terms of security.

\section{REFERENCES}

[1] Ajay Jangra, Nitin Goel, Priyanka \& Komal Bhatia, "Security Aspects in Mobile Ad Hoc Networks (MANETs): A Big Picture", International Journal of Electronics Engineering, 2(1), 2010, pp. 189-196. 
[2] Zhu, D., Mutka, M. W., \& Cen, Z. (2004, October). QoS Aware Wireless Bandwidth Aggregation (QAWBA) by Integrating Cellular and Ad-Hoc Networks. In QSHINE (pp. 156-163.

[3] Gupta, B. K., Lal, M., \& Sharma, S. C. (2005, November). Improving quality of service parameters in wireless asynchronous transfer mode network. In Mobile Technology, Applications and Systems, 2005 2nd International Conference on (pp. 1-8). IEEE.

[4] Terrill L. Frantz, Kathleen M. Carley, "A Formal Characterization of Cellular Networks", CMU-ISRI-05109, September 2005

[5] Chebrolu, K., \& Rao, R. R. (2006). Bandwidth aggregation for real-time applications in heterogeneous wireless networks. Mobile Computing, IEEE Transactions on, 5(4), 388-403.

[6] Elias Z. Tragos and George Tsiropoulos (2008) Admission Control for QoS Support in Heterogeneous 4G Wireless Networks, in IEEE Network - May/June 2008

[7] Kirubakaran, S., \& MANOHARAN, D. C. (2010). Reducing Packet Delay and Loss in Heterogeneous Mobile Wireless Networks. Global Journal of Computer Science and Technology, 9(5).

[8] Mairh, A., \& Pati, H. K. (2011, February). Quality-ofservice at data link layer in wireless cellular networks. In Proceedings of the 2011 International Conference on
Communication, Computing \& Security (pp. 162-165). $\mathrm{ACM}$

[9] Li, J., Cheng, J. H., Shi, J. Y., \& Huang, F. (2012). Brief Introduction of Back Propagation (BP) Neural Network Algorithm and Its Improvement. In Advances in Computer Science and Information Engineering (pp. 553-558). Springer Berlin Heidelberg.

[10] Al-Hamadi, H., \& Chen, R. (2013). Redundancy management of multipath routing for intrusion tolerance in heterogeneous wireless sensor networks. Network and Service Management, IEEE Transactions on, 10(2), 189203.

[11] Bui, D. H., Lee, K., Oh, S., Shin, I., Shin, H., Woo, H., \& Ban, D. (2013, December). GreenBag: energy-efficient bandwidth aggregation for real-time streaming in heterogeneous mobile wireless networks. In Real-Time Systems Symposium (RTSS), 2013 IEEE 34th (pp. 57-67). IEEE

[12] Sourish Mitra, Rafiqul Islam, (2014) A Modified Algorithmic Approach of DSDV Routing Protocol for Wireless Ad Hoc Network, IOSR Journal of Computer Engineering (IOSR-JCE) e-ISSN: 2278-0661, p- ISSN: 2278-8727Volume 14, Issue 4 (Sep. - Oct. 2013), PP 4954

[13] Bala Murali Krishna K, Madhuri S , Vanlin Sathya and Bheemarjuna Reddy Tamma, (2014) A Dynamic Link Aggregation Scheme for Heterogeneous Wireless Networks, IEEE CONECCT2014 1569825269. 